PROCEEDINGS OF THE

AMERICAN MATHEMATICAL SOCIETY

Volume 136, Number 9, September 2008, Pages 3223-3229

S 0002-9939(08)09353-2

Article electronically published on April 29, 2008

\title{
TURÁN TYPE INEQUALITIES FOR HYPERGEOMETRIC FUNCTIONS
}

\author{
ÁRPÁD BARICZ \\ (Communicated by Carmen C. Chicone) \\ Dedicated to the memory of Professor Alexandru Lupaş
}

\begin{abstract}
In this note our aim is to establish a Turán type inequality for Gaussian hypergeometric functions. This result completes the earlier result that G. Gasper proved for Jacobi polynomials. Moreover, at the end of this note we present some open problems.
\end{abstract}

\section{INTRODUCTION}

The famous result of Turán [19], established in 1950, is the following inequality:

$$
\left[P_{n+1}(r)\right]^{2}>P_{n}(r) P_{n+2}(r) \text { for all } r \in(-1,1) \text { and } n=0,1,2, \ldots,
$$

where $P_{n}$ is the Legendre polynomial, that is,

$$
P_{n}(r):=\frac{\mathrm{d}^{n}}{\mathrm{dr}^{n}}\left[\frac{\left(r^{2}-1\right)^{n}}{n ! 2^{n}}\right]={ }_{2} F_{1}\left(-n, n+1,1, \frac{1-r}{2}\right) .
$$

Here ${ }_{2} F_{1}(a, b, c, r)$ denotes the Gaussian hypergeometric function [4, p. 64], which for real numbers $a, b, c$ and $c \neq 0,-1,-2, \ldots$ has the infinite series representation

$$
{ }_{2} F_{1}(a, b, c, r):=\sum_{n \geq 0} \frac{(a)_{n}(b)_{n}}{(c)_{n}} \cdot \frac{r^{n}}{n !} \text { for all } r \in(-1,1),
$$

where $(a)_{0}=1$ for $a \neq 0$ and $(a)_{n}=a(a+1) \ldots(a+n-1)=\Gamma(a+n) / \Gamma(a)$ for each $n=1,2, \ldots$ denotes the well-known Pochhammer (or Appell) symbol. Turán's inequality established for Legendre polynomials has generated considerable interest. This classical result has been extended in several directions: ultraspherical, Laguerre and Hermite polynomials [16, Jacobi polynomials [10, 11, general class of polynomials [9], Bessel functions of the first kind [15, modified Bessel functions of the first kind [6, 13, 18, and so forth. This inequality still attracts the attention of mathematicians, and it is worth mentioning here that recently the inequality (1.1) was improved by Alzer et al. 2.

Received by the editors July 23, 2007.

2000 Mathematics Subject Classification. Primary 33C05; Secondary 26D07.

Key words and phrases. Hypergeometric function, Legendre polynomial, Jacobi polynomial, Turán inequality. 
Karlin and Szegö in their mammoth work 14 raised the question of determining the explicit range of parameters $\alpha$ and $\beta$ for which the generalized Turán inequality

$$
\left[R_{n+1}^{(\alpha, \beta)}(r)\right]^{2}>R_{n}^{(\alpha, \beta)}(r) R_{n+2}^{(\alpha, \beta)}(r)
$$

holds for all $r \in(-1,1)$ and $n=0,1,2, \ldots$, where

$$
R_{n}^{(\alpha, \beta)}(r)=P_{n}^{(\alpha, \beta)}(r) / P_{n}^{(\alpha, \beta)}(1)
$$

is the normalized Jacobi polynomial and $P_{n}^{(\alpha, \beta)}$ is the Jacobi polynomial, that is

$$
P_{n}^{(\alpha, \beta)}(r):=\frac{(\alpha+1)_{n}}{n !}{ }_{2} F_{1}\left(-n, n+\alpha+\beta+1, \alpha+1, \frac{1-r}{2}\right), \quad \alpha, \beta>-1 .
$$

Clearly we have $R_{n}^{(0,0)}(r)=P_{n}(r)$ and

$$
R_{n}^{(\alpha, \beta)}(r):={ }_{2} F_{1}\left(-n, n+\alpha+\beta+1, \alpha+1, \frac{1-r}{2}\right) .
$$

In 1962 Szegö [17] proved that (1.3) holds for all $\beta \geq|\alpha|$ and $\alpha>-1$. Gasper [10, 11 improved this result by showing that in fact (1.3) holds if and only if $\beta \geq \alpha>-1$. Now let us suppose that $\beta=0$ and let us consider the notation $F_{a}(r)={ }_{2} F_{1}(a, c-a, c, r)$, where $r \in(0,1), c=\alpha+1 \in(0,1], a=-n$ and $n=0,1,2, \ldots$ Using Gasper's result and (1.4) for $\beta=0$, we obtain that the inequality (1.3) is equivalent to

$$
\left[F_{\frac{a_{1}+a_{2}}{2}}(r)\right]^{2}>F_{a_{1}}(r) \cdot F_{a_{2}}(r),
$$

where $a_{1}=a$ and $a_{2}=a-2$. Thus it is natural to ask whenever (1.5) or its reverse holds for other values of $a$. Our main motivation to write this note was this question, which we answer in the next section. It is worth mentioning here that the positive answer - in the particular case $c=1$ - to the above question was given in [5. Theorem 2.5], which was motivated by the inequality (1.1). In fact, in Theorem 2.3 we prove a stronger statement, namely that the function $a \mapsto F_{a}(r)$ is strictly concave on $(0, c)$ for each fixed $r \in(0,1)$ and $c \in(0,1]$. This completes the result of Gasper in the case of $\beta=0$. At the end of section 2 we formulate some open problems which may be of interest for further research.

\section{A Turán type inequality FOR GAUSSIAN HYPERGEOMETRIC FUNCTIONS}

The following technical lemma improves [5, Lemma 2.2] and is one of the crucial facts in the proof of our main result.

Lemma 2.1. Let us consider the sequence of functions $f_{n}(a)=(a)_{n}(c-a)_{n}$, where $n=1,2, \ldots$ and $0 \leq a \leq c \leq 1$. For each $n=1,2, \ldots$ the following assertions are true:

(a) $f_{n}$ is positive, is increasing on $[0, c / 2]$ and is decreasing on $[c / 2, c]$;

(b) $g_{n}=f_{n} / a$ is strictly decreasing on $(0, c]$;

(c) $g_{1}=f_{1} / a$ is concave and $g_{n}=f_{n} / a$ is strictly concave on $(0, c / 2]$ for each $n \geq 2$

(d) $f_{n}$ is strictly concave on $(0, c)$. 
Proof. (a) Clearly the function $f_{1}(a)=a(c-a)$ is increasing on $[0, c / 2]$ and is decreasing on $[c / 2, c]$. Now suppose that for some $n \geq 2$ the function $f_{n}$ has the same property. Since $(\alpha)_{n+1}=(\alpha)_{n}(\alpha+n)$, one has

$$
f_{n+1}(a)=f_{n}(a) h_{n}(a), \text { where } h_{n}(a)=(a+n)(c-a+n), n=1,2, \ldots
$$

Clearly the function $h_{n}$ for each $n=1,2, \ldots$ is increasing on $[0, c / 2]$ and is decreasing on $[c / 2, c]$. Thus $f_{n+1}$ has the same property, hence by mathematical induction the required result follows.

(b) First suppose that $a \in(0, c / 2]$ and let us write $g_{n}(a)=u(a) v_{n}(a)$, where

$$
u(a)=[\Gamma(a+1) \Gamma(c-a)]^{-1} \text { and } v_{n}(a)=\Gamma(a+n) \Gamma(c-a+n) .
$$

Then clearly we have

$$
\begin{aligned}
& {[\log u(a)]^{\prime}=\psi(c-a)-\psi(a+1)<0, \quad a>0 \geq(c-1) / 2,} \\
& {\left[\log v_{n}(a)\right]^{\prime}=\psi(a+n)-\psi(c-a+n) \leq 0, \quad 0<a \leq c / 2,}
\end{aligned}
$$

where we have used the fact that the digamma function $x \mapsto \psi(x)=\Gamma^{\prime}(x) / \Gamma(x)$ is increasing on $(0, \infty)$, i.e. the gamma function is log-convex. Consequently the function $u$ is strictly decreasing and $v_{n}$ is decreasing, and thus the function $g_{n}$ is strictly decreasing, too.

Now assume that $a \in[c / 2, c]$. From part (a) $f_{n}$ is decreasing and thus $g_{n}$ is clearly strictly decreasing as a product of a decreasing and a strictly decreasing function.

(c) The function $g_{1}(a)=f_{1}(a) / a=c-a$ is concave on $(0, c / 2]$ and $g_{2}(a)=$ $f_{2}(a) / a$ is strictly concave on $(0, c / 2]$. Now suppose that $g_{n}$ is strictly concave, too, for some $n \geq 3$. From (2.2) $g_{n+1}(a)=g_{n}(a) h_{n}(a)$, and thus

$$
g_{n+1}^{\prime \prime}(a)=g_{n}^{\prime \prime}(a) h_{n}(a)+2 g_{n}^{\prime}(a) h_{n}^{\prime}(a)+g_{n}(a) h_{n}^{\prime \prime}(a)<0,
$$

because $g_{n}$ is strictly decreasing from part (b), and $h_{n}$ is increasing and strictly concave on $(0, c / 2]$. Mathematical induction implies the strict concavity of $g_{n}$.

(d) Since $f_{n}(a)=f_{n}(c-a)$, it is enough to show the strict concavity of $f_{n}$ for $a \in(0, c / 2]$. First suppose that $n=1$. Then $f_{1}(a)=a(c-a)$ is clearly strictly concave on $(0, c / 2]$. Now assume that $n \geq 2$. Because from part (c) $g_{n}$ is strictly concave, one has for each $n=2,3 \ldots$ and $a \in(0, c / 2]$

$$
g_{n}^{\prime \prime}(a)=\left(\frac{f_{n}(a)}{a}\right)^{\prime \prime}=\frac{1}{a^{3}}\left[a^{2} f_{n}^{\prime \prime}(a)-2 a f_{n}^{\prime}(a)+2 f_{n}(a)\right]<0 .
$$

From this we have $a^{2} f_{n}^{\prime \prime}(a)<2 a f_{n}^{\prime}(a)-2 f_{n}(a)$. Finally, since $g_{n}$ from part (b) is strictly decreasing on $(0, c / 2]$, we obtain that $\log g_{n}$ is strictly decreasing, too, and consequently $\left(\log \left[f_{n}(a) / a\right]\right)^{\prime} \leq 0$. From this we obtain that $a f_{n}^{\prime}(a) \leq f_{n}(a)$, and hence $f_{n}^{\prime \prime}(a)<0$. Thus the proof is complete.

Our main result of this section improves [5, Theorem 2.5].

Theorem 2.3. For $0<a<c \leq 1, r \in(0,1)$ the function $a \mapsto F_{a}(r)=$ ${ }_{2} F_{1}(a, c-a, c, r)$ is strictly sub-additive and strictly concave, consequently is strictly log-concave. In particular, for all $a_{1}, a_{2} \in(0, c)$ and $r \in(0,1)$, we have

$$
\sqrt{F_{a_{1}}(r) F_{a_{2}}(r)} \leq \frac{F_{a_{1}}(r)+F_{a_{2}}(r)}{2} \leq F_{\frac{a_{1}+a_{2}}{2}}(r) \leq F_{\frac{a_{1}}{2}}(r)+F_{\frac{a_{2}}{2}}(r),
$$

and in each of the above inequalities equality holds if and only if $a_{1}=a_{2}$. 
Proof. Using (1.2) clearly we have

$$
F_{a}(r)=\sum_{n \geq 0} \frac{f_{n}(a)}{(c)_{n} n !} r^{n},
$$

where $f_{n}(a)=(a)_{n}(c-a)_{n}$. From part (b) of Lemma 2.1 the function $a \mapsto f_{n}(a) / a$ is strictly decreasing on $(0, c)$ for each $n=1,2, \ldots$, thus clearly $f_{n}$ is strictly subadditive. From this we have that for all $a_{3}, a_{4} \in(0, c), a_{3} \neq a_{4}$ and $r \in(0,1)$

$$
F_{a_{3}+a_{4}}(r)=\sum_{n \geq 0} \frac{f_{n}\left(a_{3}+a_{4}\right)}{(c)_{n} n !} r^{n}<\sum_{n \geq 0} \frac{f_{n}\left(a_{3}\right)+f_{n}\left(a_{4}\right)}{(c)_{n} n !} r^{n}=F_{a_{3}}(r)+F_{a_{4}}(r),
$$

i.e. the function $a \mapsto F_{a}(r)$ is strictly sub-additive. Now from part (d) of Lemma 2.1 we know that $a \mapsto f_{n}(a)$ is strictly concave, thus for all $a_{1}, a_{2} \in(0, c), a_{1} \neq a_{2}$, $r \in(0,1)$ and $\lambda \in(0,1)$ we have

$$
\begin{aligned}
F_{\lambda a_{1}+(1-\lambda) a_{2}}(r) & =\sum_{n \geq 0} \frac{f_{n}\left(\lambda a_{1}+(1-\lambda) a_{2}\right)}{(c)_{n} n !} r^{n} \\
& >\sum_{n \geq 0} \frac{\lambda f_{n}\left(a_{1}\right)+(1-\lambda) f_{n}\left(a_{2}\right)}{(c)_{n} n !} r^{n}=\lambda F_{a_{1}}(r)+(1-\lambda) F_{a_{2}}(r),
\end{aligned}
$$

i.e. the function $a \mapsto F_{a}(r)$ is strictly concave, too. Finally, since the concavity is stronger than the log-concavity, the proof is complete.

We note that in fact the first and second inequalities in Theorem 2.3 may be confined as inequalities between geometric and arithmetic means. Namely, the first inequality is actually the arithmetic-geometric inequality between the values $F_{a_{1}}(r)$ and $F_{a_{2}}(r)$. These suggest the following interesting open problem:

Open problem. If $m_{1}$ and $m_{2}$ are two-variable means, i.e. for $i=1,2$ and for all $x, y, \alpha>0$ we have $m_{i}(x, y)=m_{i}(y, x), m_{i}(x, x)=x, m_{i}(\alpha x, \alpha y)=\alpha m_{i}(x, y)$ and $x<m_{i}(x, y)<y$ whenever $x<y$, then find conditions on $a_{1}, a_{2}>0$ and $c>0$ for which the inequality

$$
m_{1}\left(F_{a_{1}}(r), F_{a_{2}}(r)\right) \leq(\geq) F_{m_{2}\left(a_{1}, a_{2}\right)}(r)
$$

holds true for all $r \in(0,1)$.

The decreasing homeomorphism $\mu_{a}:(0,1) \rightarrow(0, \infty)$, defined by

$$
\mu_{a}(r):=\frac{\pi}{2 \sin \pi a} \frac{{ }_{2} F_{1}\left(a, 1-a, 1,1-r^{2}\right)}{{ }_{2} F_{1}\left(a, 1-a, 1, r^{2}\right)},
$$

where $a \in(0,1)$, is the so-called generalized Grötzsch ring function, which appears in Ramanujan's generalized modular equations (see for example [3]). This function and its particular form $\mu_{1 / 2}=\mu$ play an important role in various fields of mathematics, for example they appear in quasiconformal theory, number theory and in estimates of the hyperbolic distance (see for instance [5, Theorem 4.1]).

Now, for $0<a<c$ consider the decreasing homeomorphism $\mu_{a, c}:(0,1) \rightarrow$ $(0, \infty)$, defined by

$$
\mu_{a, c}(r):=\frac{\Gamma(a) \Gamma(c-a)}{2 \Gamma(c)} \frac{{ }_{2} F_{1}\left(a, c-a, c, 1-r^{2}\right)}{{ }_{2} F_{1}\left(a, c-a, c, r^{2}\right)},
$$


which is a natural extension of $\mu_{a}$ and is called the generalized modulus [12. In [5. Corollary 2.13] we proved that the function $a \mapsto \mu_{a}(r)$ is strictly log-convex on $(0,1)$. Since $\mu_{a, 1}=\mu_{a}$, the next result improves [5, Corollary 2.13].

Corollary 2.4. For $c \in(0,1]$ and $r \in(0,1)$ fixed the function $a \mapsto \mu_{a, c}(r)$ is strictly log-convex on $(0, c)$. In particular, $\mu_{a, c}$ satisfies the reversed Turán type inequality, that is, for all $a_{1}, a_{2} \in(0, c)$ and $r \in(0,1)$ we have

$$
\left[\mu_{\frac{a_{1}+a_{2}}{2}, c}(r)\right]^{2} \leq \mu_{a_{1}, c}(r) \mu_{a_{2}, c}(r)
$$

where equality holds if and only if $a_{1}=a_{2}$.

Proof. In [5, Theorem 2.14] we proved that the generalized complete elliptic integral 12 ]

$$
a \mapsto \mathcal{K}_{a, c}(r):=\frac{\Gamma(a) \Gamma(c-a)}{2 \Gamma(c)}{ }_{2} F_{1}\left(a, c-a, c, 1-r^{2}\right)
$$

is log-convex, whenever $0<a<\min \{c, 1\}$ and $r \in(0,1)$. On the other hand, from Theorem 2.3 we know that the function $a \mapsto{ }_{2} F_{1}\left(a, c-a, c, r^{2}\right)$ is strictly log-concave on $(0, c)$. Thus, the function $a \mapsto \mu_{a, c}(r)$ is strictly log-convex as a product of a strictly log-convex and log-convex functions.

\section{Open Problems.}

(a) Recall that from [5, Theorem 2.14] it is known that in fact the function $c \mapsto$ $\mathcal{K}_{a, c}(r)$ is log-convex, too, whenever $0<a<\min \{c, 1\}$ and $r \in(0,1)$. This suggest the following question: is the generalized modulus $\mu_{a, c}$ strictly logconvex with respect to the parameter $c$ ? Our numerical experiments suggest the validity of the following conjecture: for each $n \geq 1$ and $0<a<c$ the function $c \mapsto(c-a)_{n} /(c)_{n}$ is strictly concave. If this result were true, then this would imply that the function $c \mapsto{ }_{2} F_{1}\left(a, c-a, c, r^{2}\right)$ is strictly concave on $(a, \infty)$ and this in turn would imply that $c \mapsto \mu_{a, c}(r)$ is strictly log-convex on $(a, \infty)$.

(b) It is known that [5, Theorem 2.14] the generalized elliptic integral $\mathcal{K}_{a, c}$ is log-convex as a function of two variables with respect to variables $(a, c)$. This naturally suggest the following question: is it true that for each $n \geq 1$ and $c>a>0$ the function $f_{n}(a, c)=(a)_{n}(c-a)_{n} /(c)_{n}$ is strictly concave as a function of two variable? If this were true, then it would imply that ${ }_{2} F_{1}\left(a, c-a, c, r^{2}\right)$ is strictly concave and $\mu_{a, c}(r)$ is strictly log-convex as functions of variables $(a, c)$.

(c) A function $f$ with domain $(0, \infty)$ is said to be completely monotonic if it possesses derivatives $f^{(m)}(x)$ for each $m=0,1,2, \ldots$ and if $(-1)^{m} f^{(m)}(x) \geq$ 0 for all $x>0$. For more information on complete monotonicity the interested reader is referred to the papers [1, 20] and to the references therein. Non-negative functions with a completely monotone derivative appear in literature as Bernstein functions [7]. It is known [8] that the function

$$
x \mapsto \frac{\Gamma(x) \Gamma(x+a+b)}{\Gamma(x+a) \Gamma(x+b)}
$$

is completely monotonic on $(0, \infty)$ for each $a, b>0$. From this we have that the function

$$
c \mapsto \frac{(c-a)_{n}}{(c)_{n}}=\frac{\Gamma(c) \Gamma(c-a+n)}{\Gamma(c-a) \Gamma(c+n)}
$$


is completely monotonic, too, on $(0, \infty)$ for each $n=1,2, \ldots$ and $a<0$. On the other hand, it is easy to verify that the function $c \mapsto(c-a)_{n} /(c)_{n}$ is increasing on $(a, \infty)$ for all $n=1,2, \ldots$ and $a>0$. Thus, in view of part (a) of these open problems we may ask the following: is it true that the function

$$
x \mapsto \frac{\Gamma(x) \Gamma(x-a+b)}{\Gamma(x-a) \Gamma(x+b)}
$$

is a Bernstein function on $(a, \infty)$ for each $a, b>0$ ?

\section{ACKNOWLEDGEMENTS}

The author is grateful to the referee for his/her helpful comments and to his colleague Alexandru Kristály for his kind interest and helpful suggestions.

\section{REFERENCES}

[1] H. Alzer, and C. Berg, Some classes of completely monotonic functions, II, Ramanujan J. 11 (2006), 225-248. MR2267677 (2007k:33001)

[2] H. Alzer, S. Gerhold, M. Kauers, and A. Lupaş, On Turán's inequality for Legendre polynomials, Expo. Math. 25 (2007), 181-186. MR2319577

[3] G.D. Anderson, S.L. Qiu, M.K. Vamanamurthy and M. Vuorinen, Generalized elliptic integrals and modular equations, Pacific J. Math. 192 (2000), 1-37. MR.1741031(2001b:33027)

[4] G.E. Andrews, R. Askey, and R. Roy, Special Functions, Cambridge University Press, Cambridge, 1999. MR 1688958(2000g:33001)

[5] Á. Baricz, Turán type inequalities for generalized complete elliptic integrals, Math. Z. 256 (2007), 895-911. MR2308896

[6] Á. Baricz, Functional inequalities involving Bessel and modified Bessel functions of the first kind, Expo. Math. (2008), doi:10.1016/j.exmath.2008.01.001.

[7] C. Berg and G. Forst, Potential Theory and Locally Compact Abellian Groups, Springer, Berlin, 1975. MR0481057 (58:1204)

[8] J. Bustoz and M.E.H. Ismail, On gamma function inequalities, Math. Comp. 47 (1986), 659-667. MR856710 (87m:33002)

[9] G. Csordas and J. Williamson, On polynomials satisfying a Turán type inequality, Proc. Amer. Math. Soc. 43(2) (1974), 367-372. MR0338487 (49:3251)

[10] G. Gasper, On the extension of Turán's inequality to Jacobi polynomials, Duke Math. J. 38 (1971), 415-428. MR0276514 (43:2258)

[11] G. Gasper, An inequality of Turán type for Jacobi polynomials, Proc. Amer. Math. Soc. 32 (1972), 435-439. MR0289826 (44:7013)

[12] V. Heikkala, M.K. Vamanamurthy and M. Vuorinen, Generalized elliptic integrals, Comput. Methods Funct. Theory 9 (1) (2009), 75-109.

[13] C.M. Joshi and S.K. Bissu, Some inequalities of Bessel and modified Bessel functions, J. Austral. Math. Soc. (Series A) 50 (1991), 333-342. MR.1094928 (92d:33007)

[14] S. Karlin, G. Szegő, On certain determinants whose elements are orthogonal polynomials, J. d'Analyse Math. 8 (1960/61), 1-157, reprinted in R. Askey, ed., "Gábor Szegő Collected Papers", vol. 3, Birkhäuser, Boston, 1982, 605-761. MR0142972 (26:539)

[15] O. Szász, Inequalities concerning ultraspherical polynomials and Bessel functions, Proc. Amer. Math. Soc. 1 (1950), 256-267. MR0034902 (11:662b)

[16] G. Szegö, On an inequality of P. Turán concerning Legendre polynomials, Bull. Amer. Math. Soc. 54 (1948), 401-405. MR0023954 (9:429d)

[17] G. Szegő, An inequality for Jacobi polynomials, Studies in Math. Anal. and Related Topics, Stanford Univ. Press., Stanford, Calif. (1962), 392-398. MR0145122 (26:2657)

[18] V.K. Thiruvenkatachar and T.S. Nanjundiah, Inequalities concerning Bessel functions and orthogonal polynomials, Proc. Indian Nat. Acad. Part A 33 (1951), 373-384. MR0048635 $(14: 44 b)$ 
[19] P. Turán, On the zeros of the polynomials of Legendre, Časopis Pest. Mat. Fys. 75 (1950), 113-122. MR0041284 (12:824g)

[20] D.V. Widder, The Laplace Trasform, Princeton Univ. Press, NJ, 1941. MR0005923 (3:232d)

Faculty of Economics, Babeş-Bolyai University, RO-400591 Cluj-Napoca, Romania

E-mail address: bariczocsi@yahoo.com 